TRANSACTIONS OF THE

AMERICAN MATHEMATICAL SOCIETY

Volume 181 , July 1973

\title{
MOMENT AND BV-FUNCTIONS ON COMMUTATIVE SEMIGROUPS
}

\author{
BY \\ P. H. MASERICK
}

ABSTRACT. A general notion of variation of functions on an arbitrary commutative semigroup with identity is introduced. The concept includes Hausdorff's for the additive semigroup of nonnegative integers as well as the more recent notions introduced for semilattices. An abstract moment problem is formulated and solved.

1. Introduction. The concept of functions of bounded variation on a linearly ordered set has been generalized to a distributive lattice [1] and more recently to a semilattice (cf. [4], [6] and [7]). Here, using different techniques, we further extend this notion to commutative semigroups with identity and show that the BVfunctions characterize the "abstract moment sequences" or what we call moment functions.

Let $S$ be a commutative semigroup with identity $l$. A nontrivial homomorphism which maps $S$ into the multiplicative semigroup of nonnegative real numbers not greater than 1 will be called an exponential. We will denote the set of all exponentials on $S$ by $\exp (S)$. Equipped with the topology of simple convergence, $\exp (S)$ is a compact Hausdorff space. We now formulate an abstract moment prob. lem. Given a real-valued function $f$ on $S$, when does there exist a regular Borel measure $\mu_{f}$ on $\exp (S)$ such that $f(x)=\int_{\exp (S)} e(x) d \mu_{f}(e)$ for all $x \in S$ ? The Stone-Weierstrass theorem implies the uniqueness of the representing measure (cf. [3]), when it exists. Thus using the terminology of [9], the abstract moment problem is completely determined. Those functions on $S$ which admit representing measures will be called moment functions.

Let $e$ be an exponential on the additive semigroup $N$ of nonnegative integers. Then $e$ is a map of the form $n \rightarrow t^{n}$ where $0 \leq t \leq 1$ and $0^{0}=1$. Thus $\exp (N)$ is homeomorphic with the closed interval $[0,1]$. Hence if $S=N$, the abstract moment problem reduces to the already solved little moment problem of Hausdorff and a real-valued function on $N$ is a moment function if and only if it is a moment sequence in the classical sense (cf. [10, p. 100]).

Received by the editors November 15, 1971.

AMS (MOS) subject classifications (1970). Primary 44A50, 46A40; Secondary 46J 25.

Key words and phrases. BV-functions, semigroup, moment problem, exponential, completely monotonic function, integral representation, finite difference, vector lattice, Banach algebra, convolution measures. 
Our main result is then that the moment functions and BV-functions coincide (Theorem 4.1). Principal results contained in [4], [6] and [7] on BV-functions on semilattices follow in a new way $(\$ 5)$. Theorem 4.1 also provides a new proof of Hausdorff's characterization of a moment sequence.

2. Preliminary. Unless otherwise stated $S$ will denote a commutative multiplicative semigroup with identity $I$. For each $x \in S$ we will set $x^{0}=l$. The translate function $f_{x}$ of a real-valued function $f$ on $S$ is defined in the usual way as $f_{x}(y)=f(x y)$ for all $x, y \in S$. Successive differences of $f$ can be defined inductively by

$$
\Delta_{0} f(\circ)=f(\circ) \text { and } \Delta_{n} f\left(\circ ; x_{1}, \cdots, x_{n}\right)=\Delta_{n-1}\left(f-f_{x_{n}}\right)\left(\circ ; x_{1}, \cdots, x_{n-1}\right) .
$$

Thus $\Delta_{n} f$ is a function of one variable with increments $x_{1}, \cdots, x_{n}$. Equivalent formulations of $\Delta_{n}$ are as follows.

(i) $\Delta_{n} f\left(x ; x_{1}, \cdots, x_{n}\right)=f(x)+\sum_{k=1}^{n}(-1)^{k} \sum_{i_{1}<\cdots<i_{k}} f\left(x \prod_{j=1}^{k} x_{i_{j}}\right)$,

(ii) $\Delta_{n} f\left(x ; x_{1}, \cdots, x_{n}\right)=\Delta_{n-1} f\left(x ; x_{1}, \cdots, x_{n-1}\right)-\Delta_{n-1} f\left(x x_{n} ; x_{1}, \cdots, x_{n-1}\right)$.

We note that the $n$th difference of $f$ is independent of the order of its increments. At times it will be convenient to write $\Delta_{n} f\left(x ; x_{1}, \cdots, x_{n}\right)$ as $\Delta_{n} f\left(x ;\left\{x_{j}\right\}\right)$. Also observe that $\Delta_{n} f_{x}\left(y ; x_{1}, \cdots, x_{k}\right)=\Delta_{n} f\left(x y ; x_{1}, \cdots, x_{n}\right)$. A real-valued function $f$ on $S$ will be called completely monotonic if $\Delta_{n} f\left(x ;\left\{x_{j}\right\}\right) \geq 0$ for all choices of $x$ and $\left\{x_{j}\right\}$.

Since the abstract moment problem $f(x)=\int_{\exp (S)} e(x) d \mu_{f}(e)$ is completely determined, the vector space $E_{\infty}(S)$ of moment functions is isomorphic to the Banach algebra of all regular Borel measures on $\exp (S)$, the isomorphism being the map $f \rightarrow \mu_{f}$. Thus $E_{\infty}(S)$ is a Banach lattice with positive cone $C_{\infty}(S)$, the class of completely monotonic functions on $S$ (cf. [3]). Hence $f$ is a moment function if and only if it is the difference of two completely monotonic functions. If $f$ is a moment function we will denote its representing measure by $\mu_{f}$. An important difference formula which will be referred to without reference is

$$
\Delta_{n} f\left(x ; x_{1}, \cdots, x_{k}\right)=\int_{\exp (S)} e(x)\left[\pi_{j}\left(1-e\left(x_{j}\right)\right)\right] d \mu_{f}(e) .
$$

In referring to a Banach lattice (including the reals $R$ ), we use $\vee$ and $\wedge$ to denote the lattice operation of join and meet; $|f|$ to denote the variation $f \vee(-f)$; and $f^{+}\left(f^{-}\right)$to denote the positive (negative) variation $f \vee 0\left((-f)^{+}\right)$. To avoid confusion we will use $[a]^{A}$ to denote the absolute value of a real number $a$. Finally we establish the convention that $0^{0}=1$. 
3. The variation of functions in $E_{\infty}(S)$. For each $x \in S$ we define the realvalued function $\hat{x}$ on $\exp (S)$ by $\hat{x}(e)=e(x)$ for all $e \in \exp (S)$. Finite linear combinations $\Sigma_{i} a_{i} \hat{x}_{i}$ will then be referred to as polynomials. Note that $(x y)^{\wedge}=\hat{x} \hat{y}$ for all $x, y \in S$, so that the polynomials form an algebra. The binomial theorem implies that $\mathbb{Q}(n, x)=\left\{\left(\begin{array}{c}n \\ m\end{array}\right) \hat{x}(1-\hat{x})^{n-m} \mid m=0,1, \cdots, n\right\}$ is a partition of unity. It follows that the collection $\pi_{j} \mathbb{Q}\left(n_{j}, x_{j}\right)$ of all termwise products of the finite collection $\left\{\mathbb{A}\left(n_{j}, x_{j}\right)\right\}$ of partitions is again a partition of unity. Such a partition we will call a binomial partition of order $\left\{n_{j}\right\}$ (or of order $n$ if $n_{j}=n$ for all $j$ ) in $k$ entries. The partition $\Pi_{j=1}^{k} \mathbb{Q}\left(n_{j}, x_{j}\right)$ is then the set

$$
\left\{\pi_{j}\left(\begin{array}{c}
n_{j} \\
i
\end{array}\right)\left(\hat{x}_{j}\right)^{i}\left(1-\hat{x}_{j}\right)^{n_{j}-i} \mid i \in \pi_{j}\left(I_{n_{j}}\right)\right\}
$$

where $I_{n_{j}}=\left\{0,1, \cdots, n_{j}\right\}$ and $\pi_{j}\left(I_{n_{j}}\right)$ is the set of all maps $i \mid j \rightarrow i_{j}$ in this product space. A subset of a partition will be called a subpartition.

Let $X^{\prime} \subset S$ such that for each $x \in S$ there exists a finite subset $\left\{x_{1}, \cdots, x_{k}\right\}$ $C X^{\prime}$ with the property that $\pi_{j} x_{j}=x$. Then $X^{\prime}$ is called a generator set for $S$. Clearly $S$ is always a generator set for itself. Throughout the paper it will be assumed that $X^{\prime}$ is a generator set for $S$.

Lemmas 3.1 and 3.2 show that there are "enough" binomial partitions of unity to determine the variation of a measure on $\exp (S)$.

Lemma 3.1. Let $K_{1}$ and $K_{2}$ be disjoint compact subsets of $\exp (S)$. There exist disjoint open sets $G_{1}$ and $G_{2}$ containing $K_{1}$ and $K_{2}$ respectively and a finite subset $\left\{x_{1}, \cdots, x_{k}\right\}$ of $X^{\prime}$ sucb that for each $\epsilon(0<\epsilon<1)$ and all sufficiently large $n_{j}$, one can find a subpartition $\mathbb{Q}_{0}$ of $\pi_{j} \mathbb{A}\left(n_{j}, x_{j}\right)$ with the property that $\Sigma\left\{p \mid p \in \mathbb{C}_{0}\right\}$ is larger than $1-\epsilon$ on $G_{1}$ and smaller than $\epsilon$ on $G_{2}$.

Note that the order $\left\{n_{j}\right\}$ of the partition depends on the choice of $\epsilon$ while the entries of the partition and separating open sets do not. Moreover the partition found may be repetitious, i. e. the entries $x_{j}$ may not all be distinct.

Proof of Lemma 3.1. Let $e_{1}, e_{2} \in \exp (S)$ such that $e_{1} \neq e_{2}$. Since $X^{\prime}$ is a generator set, there exists $x \in X^{\prime}$ such that $\hat{x}\left(e_{1}\right) \neq \hat{x}\left(e_{2}\right)$. Choose $\delta>0$ such that the intervals $I\left(\delta, e_{q}\right)=\left(\hat{x}\left(e_{q}\right)-\delta, \hat{x}\left(e_{q}\right)+\delta\right)(q=1,2)$ are disjoint. Set $G_{1}\left(e_{1}, e_{2}\right)=\left\{e \in \exp (S)|| \hat{x}(e)-\hat{x}\left(e_{1}\right) \mid<\delta / 2\right\}$ and $G_{2}\left(e_{1}, e_{2}\right)=\{e \in \exp (S)|| \hat{x}(e)-$ $\left.\hat{x}\left(e_{2}\right) \mid<\delta / 2\right\}$. Clearly $G_{1}\left(e_{1}, e_{2}\right)$ and $G_{2}\left(e_{1}, e_{2}\right)$ are disjoint open sets containing $e_{1}$ and $e_{2}$ respectively. Denote the characteristic function of the interval $I\left(\delta, e_{1}\right)$ by $\chi$. Uniform continuity of $\chi$ on $I\left(\delta / 2, e_{1}\right) \cup \cdot I\left(\delta / 2, e_{2}\right)$ implies that the sequence

$$
\Re_{n}(\chi)=\sum_{i} \chi\left(\frac{i}{n}\right)\left(\begin{array}{l}
n \\
i
\end{array}\right) t^{i}(1-t)^{n-i}
$$


of Bernstein polynomials converges uniformly to $\chi$ on this union [5, p. 6]. Thus for sufficiently large $n$, the subpartition $\left\{\chi(i / n)\left(\begin{array}{c}n \\ i\end{array}\right) \hat{x}^{i}(1-\hat{x})^{n-i}\right\}$ can be made arbitrarily close to 1 on $G_{1}\left(e_{1}, e_{2}\right)$ and arbitrarily close to 0 on $G_{2}\left(e_{1}, e_{2}\right)$. That is, we have proved the assertion for the special case where $K_{1}$ and $K_{2}$ are both singletons. Moreover, for this special case, the required partition need only have one entry.

Let $e_{1} \notin K_{2}$. By compactness the collection $\left\{G_{2}\left(e_{1}, e_{2}\right) \mid e_{2} \in K_{2}\right\}$ admits a finite subcovering $\left\{G_{2}\left(e_{1}, e_{2 j}\right) \mid j=1,2, \cdots, k\right\}$ of $K_{2}$. For each $j$ let $x_{1,2 j}$ denote the entry in $X^{\prime}$ of the partition found in the above paragraph for the pair $G_{1}\left(e_{1}, e_{2 j}\right), G_{2}\left(e_{1}, e_{2 j}\right)$. The partition found is then $C\left(n_{j}, x_{1,2 j}\right)$ and its corresponding subpartition $\mathbb{a}_{0}\left(n_{j}, x_{1,2 j}\right)$ has the property that

$$
\sum\left\{p \mid p \in \mathbb{A}_{0}\left(n_{j}, x_{1,2 j}\right)\right\} \begin{cases}>(1-\epsilon)^{1 / k} & \text { on } G_{1}\left(e_{1}, e_{2 j}\right) \\ <\epsilon & \text { on } G_{2}\left(e_{1 j}, e_{2}\right)\end{cases}
$$

for sufficiently large $n_{j}$. If we set $G_{1}\left(e_{1}, K_{2}\right)=\bigcap_{j} G_{1}\left(e_{1}, e_{2 j}\right)$ and $G_{2}\left(e_{1}, K_{2}\right)$ $=\bigcup_{j} G_{2}\left(e_{1}, e_{2 j}\right)$ then

$$
\sum\left\{p \mid p \in \pi_{j} \mathbb{Q}_{0}\left(n_{j}, x_{1,2 j}\right)\right\} \begin{cases}>1-\epsilon & \text { on } G_{1}\left(e_{1}, K_{2}\right) \\ <\epsilon & \text { on } G_{2}\left(e_{1}, K_{2}\right)\end{cases}
$$

and hence

$$
\sum\left\{p \mid p \in\left[\prod_{j} \mathfrak{a}\left(n_{j}, x_{1,2 j}\right)\right] \backslash \prod_{j} \mathfrak{A}_{0}\left(n_{j}, x_{1,2 j}\right)\right\} \begin{cases}<\epsilon & \text { on } G_{1}\left(e_{1}, K_{2}\right) \\ >1-\epsilon & \text { on } G_{2}\left(e_{1}, K_{2}\right)\end{cases}
$$

that is we have proved the assertion for the case where either $K_{1}$ or $K_{2}$ is a singleton. The final assertion follows by using the compactness of $K_{1}$, as well as the last formula and repeating the above argument.

Lemma 3.2. Let $\mathbb{I}$ denote an arbitrary nonrepetitious binomial partition of degree $n$ with entries in $X^{\prime}$ and $E$ denote a Borel subset of $\exp (S)$. If $\mu$ is a regular signed measure on $\exp (S)$ and $|\mu|$ is the variation of $\mu$ then

$$
|\mu|(E)=\sup _{\boldsymbol{P}} \sum_{p \in \mathbb{P}}\left[\int_{E} p d \mu\right]^{A} \text {. }
$$

Proof. From the Hahn decomposition we can find a $\mu$-positive subset $A$ of $E$ such that $B=E \backslash A$ is $\mu$-negative. If $Q$ is an arbitrary binomial partition then

$$
|\mu|(E)=\sum_{p \in \mathbb{Q}} \int_{A} p d \mu-\sum_{p \in \mathbb{Q}} \int_{B} p d \mu \geq \sum_{p \in \mathbb{Q}}\left[\int_{E} p d \mu\right]^{A}
$$


so that

$$
|\mu|(E) \geq \sup _{\mathbb{Q}} \sum_{p \in \mathbb{Q}}\left[\int_{E} p d \mu\right]^{A} .
$$

To reverse the inequality, we use regularity of $\mu$ to approximate $A$ and $B$ from within by compact subsets $K_{1}$ and $K_{2}$. Lemma 3.1 implies the existence of a possibly repetitious partition $\mathbb{Q}=\Pi_{j} \mathfrak{Q}\left(n_{j}, x_{j}\right)$ such that $\Sigma_{p \in Q}\left[\int_{E} p d \mu\right]^{A}$ approximates $|\mu|(E)$ from below. Moreover by taking the degree of $\mathbb{A}$ large enough we can insure that $\left\{\sum n_{j} \mid x_{j}=x_{l}\right\}$ is the same integer, say $n$, for each $l=1, \cdots, k$. A typical function in $\mathbb{T}$ is of the form

$$
\left(\begin{array}{l}
n_{1} \\
i_{1}
\end{array}\right) \cdots\left(\begin{array}{l}
n_{k} \\
i_{k}
\end{array}\right)\left(\hat{x}_{1}\right)^{i_{1}} \ldots\left(\hat{x}_{k}\right)^{i_{k}}\left(1-\hat{x}_{1}\right)^{n_{1}-i_{1}} \ldots\left(1-\hat{x}_{k}\right)^{n_{k}-i_{k}} .
$$

If $i$ is repetitious, then by adding exponents of repetitious factors, (i) can be expressed so that no factor is duplicated. If we now group those functions in $\mathbb{A}$ whose corresponding factors have the same degree, then the set of sums of these groups gives another partition $\therefore$ of unity. The identity

$$
\left(\begin{array}{c}
m_{1}+\cdots+m_{p} \\
l
\end{array}\right)=\sum\left\{\left.\left(\begin{array}{l}
m_{1} \\
l_{1}
\end{array}\right) \cdots\left(\begin{array}{c}
m_{p} \\
l_{p}
\end{array}\right)\right|_{1}+\cdots+l_{p}=l\right\}
$$

implies that $\mathbb{A}^{\prime}$ is also a binomial partition. Moreover $\Phi^{\prime}$ is nonrepetitious and of degree $n$. The assertion follows since

$$
\sum_{p \in \mathbb{Q}}\left[\int_{E} p d \mu\right]^{A}=\sum_{p \in \mathbb{Q}^{\prime}}\left[\int_{E} p d \mu\right]^{A} .
$$

Let $i \mid j \rightarrow i_{j}$ be a function in the product space $\Pi_{j}\left(I_{n_{j}}\right),\left\{x_{j}\right\}$ a finite sequence in $S$ and $f$ a real-valued function on $S$. We introduce the notation $\Delta f\left(\left\{n_{j}\right\},\left\{x_{j}\right\}, i\right)$ to abbreviate

$$
\prod_{j}\left(\begin{array}{c}
n \\
i_{j}
\end{array}\right) \Delta f\left(\prod_{j}\left(x_{j}\right)^{i}{ }_{j} \frac{x_{1}, \cdots, x_{1}}{n_{k}-i_{1}}, \cdots, \frac{x_{k}, \cdots, x_{k}}{n_{k}-i_{k}}\right)
$$

where each increment $x_{j}$ appears $n_{j}-i_{j}$ times. If $n_{j}=n$ for each $j$ then we will replace $\left\{n_{j}\right\}$ by $n$. Clearly the above difference does not depend on the order of the sequence $\left\{x_{j}\right\}$. Thus if $X$ is any finite subset of $S$ then $\Delta f\left(\left\{n_{j}\right\}, X, i\right)$ is well defined and the increments are not repetitious. Note that if

$$
p\left(\left\{n_{j}\right\},\left\{x_{j}\right\}, i\right)=\prod_{j}\left[\left(\begin{array}{c}
n_{j} \\
i_{j}
\end{array}\right)\left(\hat{x}_{j}\right)^{i}{ }_{j}\left(1-\hat{x}_{j}\right)^{n_{j}-i_{j}}\right]
$$

and if $\mu_{j}$ is the representing measure for $f \in E_{\infty}(S)$ then $\$ 2$ (iii) implies $\Delta f\left(\left\{n_{j}\right\},\left\{x_{j}\right\}, i\right)=\int_{\exp (S)} p\left(\left\{n_{j}\right\},\left\{x_{j}\right\}, i\right) d \mu_{f}$.

For each $f \in E_{\infty}(S)$ we define the total variation $\|f\|$ as the total variation 
$\left\|\mu_{f}\right\|$ of the representing measure $\mu_{f}$. The following theorem follows easily from Lemma 3.2.

Theorem 3.3. Let $f \in E_{\infty}(S)$ and $X$ denote an arbitrary finite subset of $X^{\prime}$. Then

$$
\|f\|=\sup _{(n, X)} \sum_{i}[\Delta f(n, X, i)]^{A} .
$$

Recall that the variation $|f|$, positive variation $f^{+}$and negative variation $f^{-}$of $f$ in a vector lattice $E$ are defined by $|f|=f \vee(-f), f^{+}=f \vee(-f)$ and $f^{-}=(-f)^{+}$. Since the map $f \rightarrow \mu_{f}$ is a lattice isomorphism we must have $|f|(x)=$ $\int \hat{x} d\left|\mu_{f}\right|,\left(f^{+}\right)(x)=\int \hat{x} d \mu_{f}^{+}$and $\left(f^{-}\right)(x)=\int \hat{x} d \mu_{f}^{-}$.

Corollary 3.4. If $f \in E_{\infty}(S)$ then $|f|(x), f^{+}(x)$ and $f^{-}(x)$ are given by

$$
\sup _{(n, X)} \sum\left[\Delta f_{x}(n, X, i)\right]^{*}
$$

where * is either $A,+$ or - respectively.

Proof. Since the representing measure for the translate $f_{x}$ of $f \in E_{\infty}(S)$ is clearly $\hat{x} d \mu_{f}$ and since $\hat{x}$ is nonnegative we must have $\left|f_{x}\right|(y)=\int \hat{y}\left(\hat{x} d\left|\mu_{f}\right|\right)$ so that $|f|(x)=\int \hat{x} d\left|\mu_{f}\right|=\left|f_{x}\right|(I)=\left\|f_{x}\right\|$. To verify the formula with $*=+$ we note that the binomial theorem implies

$$
\begin{aligned}
\sum_{i} \Delta f\left(x,\left\{x_{j}\right\}, i\right) & =\sum_{i} \int \hat{x} \prod_{j}\left[\left(\begin{array}{c}
n \\
i_{j}
\end{array}\right)\left(\hat{x}_{j}\right)^{i}{ }^{j}\left(1-\hat{x}_{j}\right)^{n-i_{j}}\right] d \mu_{f} \\
& =\int \hat{x} \sum_{i} \prod_{j}\left[\left(\begin{array}{c}
n \\
i_{j}
\end{array}\right)\left(\hat{x}_{j}\right)^{i}\left(1-\hat{x}_{j}\right)^{n-i_{j}}\right] d \mu_{f} \\
& =\int \hat{x} \prod_{j}\left[\sum_{l=1}^{n}\left(\begin{array}{c}
n \\
i
\end{array}\right) \hat{x}_{j}^{l}\left(1-\hat{x}_{j}\right)^{n-l}\right] d \mu_{f}=\int \hat{x} d \mu_{f}=f(x) .
\end{aligned}
$$

Thus the first assertion implies

$$
\begin{aligned}
f^{+}(x)= & 1 / 2[f(x)+|f|(x)] \\
= & \sup _{(n, X)} \sum_{i} 1 / 2\left(\Delta f(n, X, i)+[\Delta f(n, X, i)]^{A}\right) \\
= & \sup _{(n, X)} \sum_{i}[\Delta f(n, X, i)]^{+} .
\end{aligned}
$$

The assertion for $*=-$ follows from the assertion for $*=+$.

The formula for $*=+$ or - allows us to express a function $f \in E_{\infty}(S)$ as the difference $f^{+}-f^{-}$of two completely monotonic functions while the formula for $*=A$ gives a method of computing the join and meet of any two functions in $E_{\infty}(S)$. 
4. Functions of bounded variation. As in $\$ 3$, we let $X$ denote an arbitrary finite subset of a fixed generator set $X^{\prime}$. We define the total variation of an arbitrary real-valued function $f$ on $S$ by $\|f\|=\sup _{(n, X)} \Sigma_{i}[\Delta f(n, X, i)]^{A}$. The consistency of this definition for functions in $E_{\infty}(S)$ is then clear from Theorem 3.3. If we denote the set of all functions on $S$ which have finite variation by $\mathrm{BV}(S)$ then we may state our main result as follows.

Theorem 4.1. $\mathrm{BV}(S)=E_{\infty}(S)$.

From Theorem 3.3 we need only prove $\mathrm{BV}(S) \subset E_{\infty}(S)$. The proof follows after Lemma 4.5 .

Lemma 4.2. If $\left\{x_{j}\right\}$ is a finite sequence in $X^{\prime}$ and $n_{j}>m_{j}$ then

$$
\sum_{i}\left[\Delta f\left(\left\{n_{j}\right\},\left\{x_{j}\right\}, i\right)\right]^{*} \geq \sum_{i}\left[\Delta f\left(\left\{m_{j}\right\},\left\{x_{j}\right\}, i\right)\right]^{*}
$$

where * is either $A,+$ or - .

Proof. All three assertions will be proved simultaneously since the only property we use of $*$ is the triangle inequality, $[a-b]^{*} \geq[a]^{*}-[b]^{*}$. By induction, it is sufficient to prove

$$
\sum_{i}\left[\Delta f\left(\left\{n_{1}, \cdots, n_{l}+1, \cdots, n_{k}\right\},\left\{x_{j}\right\}, i\right)\right]^{*} \geq \sum_{i}\left[\Delta f\left(\left\{n_{j}\right\},\left\{x_{j}\right\}, i\right)\right]^{*} .
$$

Moreover, since differencing does not depend on the order of the increments we must have

$$
\begin{aligned}
\sum\left\{\left[\Delta f\left(\left\{n_{j}\right\},\left\{x_{j}\right\}, i\right)\right]^{*} \mid i \in \prod_{j}\left(I_{n_{j}}\right)\right\} \\
\left.=\sum\left\{\left[\Delta f\left\{n_{a(j)}\right\},\left\{x_{a(j)}\right\}, i\right)\right]^{*} \mid i \in \prod_{j}\left(l_{n a(j)}\right)\right\}
\end{aligned}
$$

where $\alpha$ is any permutation of the first $k$ natural numbers. Thus we may assume $l=k$ and will complete the proof by showing

$$
\begin{aligned}
& \sum\left\{\left(\begin{array}{c}
n_{1} \\
i_{1}
\end{array}\right) \cdots\left(\begin{array}{c}
n_{k} \\
i_{k}
\end{array}\right)\left[\Delta f\left(x_{1}^{i_{1}} \ldots x_{k}^{i_{k}} ; \frac{x_{1}, \cdots, x_{1}}{n_{1}-i_{k}}, \cdots, \frac{x_{k}, \cdots, x_{k}}{n_{k}-i_{k}}\right)\right]^{*} \mid i_{k}=0,1, \cdots, n_{k}\right\} \\
& \leq \sum\left\{\left(\begin{array}{c}
n_{1} \\
i_{1}
\end{array}\right) \cdots\left(\begin{array}{c}
n_{k}+1 \\
i_{k}
\end{array}\right)\left[\Delta f\left(x_{1}^{i_{1}} \ldots x_{k}^{i_{k}} ; \frac{x_{1}, \cdots, x_{1}}{n_{1}-i_{1}}, \cdots, \frac{x_{k}, \cdots, x_{k}}{\left(n_{k}+1\right)-i_{k}}\right)\right]\right. \\
& \left.i_{k}=0,1, \cdots, n_{k}+1\right\} \text {. }
\end{aligned}
$$

Since (i) follows easily from the triangle inequality for $n_{k}=0$ we will assume $n_{k}>0$ and show $A_{p} \geq A_{p+1}$ where 


$$
A_{p}=\sum\left\{( \begin{array} { c } 
{ n _ { 1 } } \\
{ i _ { 1 } }
\end{array} ) \cdots ( \begin{array} { c } 
{ n _ { k } } \\
{ i _ { k } }
\end{array} ) \left[\Delta f \left(x_{1}^{i_{1}}, \ldots x_{k}^{i_{k}}\right.\right.\right.
$$

$$
\begin{aligned}
& \left.\left.\left.\frac{x_{1}, \cdots, x_{1}}{n_{1}-i_{1}}, \cdots, \frac{x_{k}, \cdots, x_{k}}{n_{k}-i_{k}}\right)\right]^{*} i_{k}=0,1, \cdots, p\right\}
\end{aligned}
$$

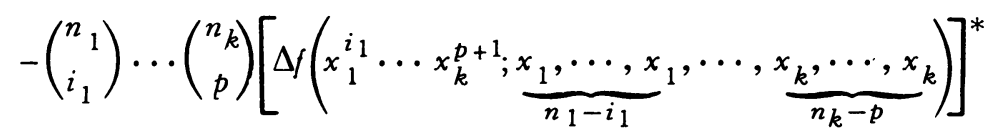

$$
\begin{aligned}
& +\sum\left\{\left(\begin{array}{c}
n_{1} \\
i_{1}
\end{array}\right) \cdots\left(\begin{array}{c}
n_{k}+1 \\
i_{k}
\end{array}\right)\left[\Delta f\left(x_{1}^{i_{1}} \ldots x_{k}^{i_{k}} ; x_{\frac{1}{n_{1}-i_{1}}}, \ldots, x_{1}, \cdots, x_{\frac{k}{\left(n_{k}+1\right)-i_{k}}}, \ldots, x_{k}\right)\right]^{*}\right. \\
& \left.i_{k}=p+1, \cdots, n_{k}+1\right\} \text {. }
\end{aligned}
$$

The last two expressions in (ii) may be rewritten as

$$
\begin{aligned}
& \left(\begin{array}{c}
n_{1} \\
i_{1}
\end{array}\right) \cdots\left(\begin{array}{c}
n_{k-1} \\
i_{k-1}
\end{array}\right)\left[\left(\begin{array}{c}
n_{k}+1 \\
p+1
\end{array}\right)-\left(\begin{array}{c}
n_{k} \\
p
\end{array}\right)\right]\left[\Delta f\left(x_{1}^{i_{1}} \cdots x_{k}^{p+1} ; \frac{x_{1}, \cdots, x_{1}}{n_{1}-i_{1}}, \cdots, \frac{x_{k}, \cdots, x_{k}}{n_{k}-p}\right)\right]^{*} \\
& +\sum\left\{\left(\begin{array}{l}
n_{1} \\
i_{1}
\end{array}\right) \cdots\left(\begin{array}{c}
n_{k}+1 \\
i_{k}
\end{array}\right)\left[\Delta f\left(x_{1}^{i_{1}} \ldots x_{k}^{i_{k}} ; \frac{x_{1}, \cdots, x_{1}, \cdots, x_{k}, \cdots, x_{k}}{n_{1}-i_{1}}\right)\right]^{*} \mid\right. \\
& \left.i_{k}=p+2, \cdots, n_{k}+1\right\} \text {. }
\end{aligned}
$$

Recall that

$$
\left(\begin{array}{c}
n_{k}+1 \\
p+1
\end{array}\right)-\left(\begin{array}{c}
n_{k} \\
p
\end{array}\right)=\left(\begin{array}{c}
n_{k} \\
p+1
\end{array}\right) \text { for } p=0,1, \cdots, n_{k}-1
$$

Thus after differencing once and using the triangle inequality $\left([a-b]^{*} \geq[a]^{*}-[b]^{*}\right)$, the first expression (iii) is seen to be larger than or equal to

$$
\left(\begin{array}{c}
n_{1} \\
i_{1}
\end{array}\right) \cdots\left(\begin{array}{c}
n_{k-1} \\
i_{k-1}
\end{array}\right)\left(\begin{array}{c}
n_{k} \\
p+1
\end{array}\right)\left[\Delta f\left(x_{1}^{i_{1}} \cdots x_{k}^{p+1} ; \frac{x_{1}, \cdots, x_{1}}{n_{1}-i_{1}}, \cdots, \frac{x_{k}, \cdots, x_{k}}{n_{k}-(p+1)}\right)\right]^{*}
$$

(iv)

$$
-\left(\begin{array}{c}
n_{1} \\
i_{1}
\end{array}\right) \cdots\left(\begin{array}{c}
n_{k-1} \\
i_{k-1}
\end{array}\right)\left(\begin{array}{c}
n_{k} \\
p+1
\end{array}\right)\left[\Delta f\left(x_{1}^{i_{1}} \cdots x_{k}^{p+2} ; \frac{x_{1}, \cdots, x_{1}, \cdots, \frac{x_{k}, \cdots, x_{k}}{n_{1}-i_{1}}}{n_{k}-(p+1)}\right)\right]^{*} \text {. }
$$

That $A_{p} \geq A_{p+1}$ for $p<n_{k}$ now follows upon including the first expression in 
(iv) under the first summation in (ii) and replacing the last two expressions in (ii) by the second formulas in both (iv) and (iii). Thus we have $A_{n_{k}} \leq A_{n_{k}-1} \leq$ $\cdots \leq A_{0}$. Since $A_{n_{k}}$ is the first summation in (i) we need only show that $A_{0}$ is no larger than the second summation. But this fact follows easily by applying the triangle inequality and formula $\$ 2$ (ii) to the first two terms which occur in the definition of $A_{0}$.

Remark. If we let $n_{k}=0$ then

$$
\sum\left\{\left[\Delta f\left(\left\{n_{j}\right\},\left\{x_{j}\right\}, i\right)\right]^{*} \mid i \in \prod_{j=1}^{k}\left(I_{n_{j}}\right)\right\}=\sum\left\{\left[\Delta f\left(\left\{n_{j}\right\},\left\{x_{j}\right\}, i\right)\right]^{*} \mid i \in \prod_{j=1}^{k-1}\left(I_{n_{j}}\right)\right\} .
$$

Thus the lemma implies that expressions of the form

$$
\sum\left\{\left[\Delta f\left(n ;\left\{x_{j}\right\}, i\right)\right]^{*} \mid i \in\left(I_{n}\right)^{k}\right\}
$$

increase with both $k$ and $n$. In particular, this means we can replace the suprema $\sup _{(n, X)}$ in Theorems 3.3 and 4.1 by $\lim _{(n, X)}$, where $\{(n, X)\}$ is made into a directed set by defining $(n, X) \geq(m, X)$ whenever $n \geq m$ and $X \supset Y$.

The next lemma shows how duplicate entries can be eliminated.

Lemma 4.3. Let $\left\{x_{j} \mid j=1, \because,(k-1)\right\} \subset S, x_{k}=x_{k-1}, m_{j}=n_{j}$ for $j=1,2$, $\cdots,(k-2)$ and $m_{k-1}=n_{k-1}+n_{k}$. Then for every real-valued function $f$ on $S$

(i) $\sum\left\{\left[\Delta f\left(\left\{n_{j}\right\},\left\{x_{j}\right\}, i\right)\right]^{A} \mid i=\prod_{j=1}^{k}\left(I_{n_{j}}\right)\right\}=\sum\left\{\left[\Delta f\left(\left\{m_{j}\right\},\left\{x_{j}\right\}, i\right)\right]^{A} \mid i \in \prod_{j=1}^{k-1}\left(I_{m_{j}}\right)\right\}$. Consequently,

(ii) $\sup _{(n, X)} \sum_{i}[\Delta f(n, X, i)]^{A}=\sup _{\left(n,\left\{x_{j}\right\}\right)} \sum_{i}\left[\Delta f\left(n,\left\{x_{j}\right\},\{i\}, i\right)\right]^{A}$ for $X,\left\{x_{j}\right\} \subset X^{\prime}$.

Proof. The equality in (i) is established by grouping and summing all differences on the left whose increment $x_{k}=x_{k-1}$ has the same number of repetitions and then applying the identity

$$
\left(\begin{array}{c}
q_{1}+\cdots+q_{p} \\
l
\end{array}\right)=\sum\left\{\left(\begin{array}{c}
q_{1} \\
l_{1}
\end{array}\right) \cdots\left(\begin{array}{c}
q_{p} \\
l_{p}
\end{array}\right) \mid l_{1}+\cdots+l_{p}=l\right\}
$$

As in $\$ 3$, we define $|f|(x)=\left\|f_{x}\right\|$.

Lemma 4.4. If $x \in S$, and $y \in X^{\prime}$ then $\Delta_{1}|f|(x ; y) \geq 0$.

Proof. Let $x_{1}, x_{2}, \cdots, x_{k} \in X^{\prime}$ and for notational convenience set $y=x_{k+1}$, $x_{j}=n$ for $j=1,2, \cdots, k$ and $n_{k+1}=1$. Then from Lemma 4.2 we have 


$$
\begin{aligned}
\sum\{ & {\left.\left[\Delta f_{x}\left(n,\left\{x_{j}\right\}, i\right)\right]^{A} \mid i \in\left(I_{n}\right)^{k+1}\right\} } \\
\geq & \sum\left\{\left[\Delta f_{x}\left(\left\{n_{j}\right\},\left\{x_{j}\right\}, i\right)\right]^{A} \mid i \in \prod_{j=1}^{k+1}\left(I_{n_{j}}\right)\right\} \\
= & \sum\left\{\prod_{j}\left(\begin{array}{l}
n \\
i_{j}
\end{array}\right)\left[\Delta f_{x}\left(x_{j}^{i_{j}} ; \frac{x_{1}, \cdots, x_{1}, \cdots, x_{k}, \cdots, x_{k}, x_{k+1}}{n-i_{1}}\right)\right]^{A} \mid i \in\left(I_{n}\right)^{k}\right\} \\
& +\sum\left\{\prod_{j}\left(\begin{array}{c}
n \\
i_{j}
\end{array}\right)\left[\Delta f_{x x_{k+1}}\left(x_{j}^{i_{j}} ; \frac{x_{1}, \cdots, x_{1}}{n-i_{1}}, \cdots, \frac{x_{k}, \cdots, x_{k}}{n-i_{k}}\right)\right]^{A} \mid i \in\left(I_{n}\right)^{k}\right\}
\end{aligned}
$$

If we drop the first term to the right of equality we get

$$
\sum\left\{\left[\Delta_{x}\left(n ;\left\{x_{j}\right\}, i\right)\right]^{A} \mid i \in\left(I_{n}\right)^{k+1}\right\} \geq \sum\left\{\left[\Delta_{x y}\left(n,\left\{x_{j}\right\}, i\right)\right]^{A} \mid i \in\left(I_{n}\right)^{k}\right\} .
$$

It now follows from Lemmas 4.2 and 4.3 that $\left\|f_{x}\right\| \geq\left\|f_{x y}\right\|$ and the proof is complete.

Lemma 4.5. If $\|f\|<\infty$ and $x \in X^{\prime}$ then $\left|f-f_{x}\right|=|f|-\left|f_{x}\right|$.

Proof. Since $\left[\Delta(f+g)_{x}(n, X, i)\right]^{A} \leq\left[\Delta f_{x}(n, X, i)\right]^{A}+\left[\Delta g_{x}(n, X, i)\right]^{A}$, we see that $|0|$ satisfies the triangle inequality. Thus $\left|f-f_{x}\right| \geq|f|-\left|f_{x}\right|$. To reverse this inequality we note that Lemma 4.4 implies $\left\|f_{x}\right\|<\infty$ so that $\left\|f-f_{x}\right\| \leq$ $\|f\|+\left\|f_{x}\right\|<\infty$. Let $\epsilon>0$ and $y \in S$ be arbitrarily chosen. From Lemma 4.2, we can find $x_{1}, x_{2}, \cdots, x_{k} \in S$ and $n$ such that

$$
\left|f-f_{x}\right|(y) \leq \sum\left\{\left[\Delta\left(f-f_{x}\right)\left(n,\left\{x_{j}\right\}, i \cdot\right)\right]^{A} \mid i \in\left(I_{n}\right)^{k}\right\}+\epsilon / 2
$$

and

$$
\left|f_{x}\right|(y) \leq \sum\left\{\left[\Delta f_{x y}\left(n,\left\{x_{j}\right\}, i\right)\right]^{A} \mid i \in\left(I_{n}\right)^{k}\right\}+\epsilon / 2 .
$$

For convenience set $x=x_{k+1}$. From the definition of difference given in $\$ 2$ the summation in (i) can be rewritten as

$$
\sum_{i} \Pi_{j}\left(\begin{array}{l}
n \\
i_{j}
\end{array}\right)\left[\Delta f_{y}\left(\prod_{j}\left(x_{j}\right)^{i_{j}} ; \frac{x_{1}, \cdots, x_{1}}{n-i_{1}}, \cdots, \frac{x_{k}, \cdots, x_{k}, x_{k+1}}{n-i_{k}}\right)\right]^{A}
$$

$$
+\sum_{i}\left[\Delta f_{y x_{k+1}}\left(n ;\left\{x_{j}\right\}, i\right)\right]^{A}-\sum_{i}\left[\Delta f_{y x_{k+1}}\left(n,\left\{x_{j}\right\}, i\right)\right]^{A},
$$

where all three summations range over $i \in\left(I_{n}\right)^{k}$. If we let $n_{j}=n$ for $j=1, \cdots$, $k$ and $n_{k+1}=1$ then the first two terms of (iii) can be grouped and expressed as

$$
\sum\left\{\left[\Delta f_{y}\left(\left\{n_{j}\right\},\left\{x_{j}\right\}, i\right)\right]^{A} \mid i \in \prod_{j=1}^{k+1}\left(I_{n_{j}}\right)\right\} \text {. }
$$


Since Lemma 4.2 implies this latter expression is dominated by

$$
\sum\left\{\left[\Delta f_{y}\left(n,\left\{x_{j}\right\}, i\right)\right]^{A} \mid i \in\left(I_{n}\right)^{k+1}\right\}
$$

we apply (ii) to get

$$
\begin{aligned}
\left|f-f_{x}\right|(y)-\epsilon / 2 & \leq \sum\left\{\left[\Delta f_{y}\left(n,\left\{x_{j}\right\}, i\right)\right]^{A} \mid i \epsilon\left(I_{n}\right)^{k+1}\right\}-\left|f_{x}\right|(y)+\epsilon / 2 \\
& \leq|f|(y)-\left|f_{x}\right|(y)+\epsilon / 2
\end{aligned}
$$

and the proof is complete.

Lemma 4.6. If $f$ is a real-valued function on $S$ such that $\Delta_{n} f\left(x ; x_{1}, \cdots, x_{n}\right)$ $\geq 0$ whenever $x_{j} \in X^{\prime}$ then $f$ is completely monotonic.

Lemma 4.6 can of course be sharpened by deriving a specific formula for the general difference. For example if $S=(N,+)$ and $X^{\prime}=\{1\}$ then the following formula can easily be checked by induction:

$$
\Delta_{k} f\left(\circ ; n_{1}, \cdots, n_{k}\right)=\sum\left\{\Delta_{k} f_{i_{1}+\cdots+i_{k}}(\circ ; 1, \cdots, 1) \mid i \in \prod_{j}\left(I_{n_{j}}\right)\right\} \text {. }
$$

For general $S$ we find it more convenient to argue analytically as follows.

Proof of Lemma 4.6. Let $C(S)$ denote the set of all functions $f$ which satisfy the above property. Then $C(S)$ is a convex cone which contains $C_{\infty}(S)$ and is closed in the topology of pointwise convergence. Since $0 \leq \Delta_{1} f\left(\Pi_{j}\left(x_{j}\right)^{i} j_{j} x_{k}\right)$ for $x_{1}, \cdots, x_{k}, \cdots, x_{n} \in X^{\prime}$ implies $f\left(\Pi_{j}\left(x_{j}\right)^{i_{j}}\right) \geq f\left[\left(\Pi_{j}\left(x_{j}\right)^{i_{j}}\right) x_{k}\right]$, it follows inductively that $f\left(\Pi_{j}\left(x_{j}\right)^{i}\right) \geq\left(\Pi_{j}\left(x_{j}\right)^{i j+l_{j}}\right)$ so that $\Delta_{1} f(x ; y) \geq 0$ for all $x, y \in$ $S$. Thus the set $B=\{f \in C(S) \mid f(1)=1\}$ is a base for $C(S)$. Note that $B$ is compact and convex. An easy computation shows that $f$ and $f-f_{x}$ are in $C(S)$ whenever $x \in X^{\prime}$. Thus if $f$ is an extreme point of $B$ we must have $a f=f_{x}$ for some nonnegative $\alpha$ and all $x \in X^{\prime}$. Evaluation at $I$ implies $\alpha=f(x)$ so that $f(x) f(y)=$ $f(x y)$ for all $x, y \in X^{\prime}$. Since $X^{\prime}$ is a generator set for $S$ we see that the exponentials on $S$ contain the extreme points of $B$. However the exponentials are in $C_{\infty}(S)$, so that the KreYn-Milman theorem implies $B$ and hence $C(S)$ is contained in $C_{\infty}(S)$.

Proof of Theorem 4.1. As mentioned earlier we need only show BV(S)C $E_{\infty}(S)$. Let $x \in X^{\prime}$ and observe that $|f|(y) \geq \Sigma\left\{\left[1 f_{y}(I,\{x\}, i)\right]^{A} \mid i \in I_{1}\right\}=$ $\left[\Delta_{0} f_{y}(x)\right]^{A}+\left[\Delta_{1} f_{y}(I ; x)\right]^{A} \geq[f(y)]^{A}$. Thus if we define $f^{+}=1 / 2(|f|+f)$ and $f^{-}=(-f)^{+}$for all $f \in \mathrm{BV}(S)$, then $\Delta_{0} f^{+}=f^{+} \geq 0$. Since $f=f^{+}-f^{-}$we need only show that $f^{+} \in C_{\infty}(S)$ whenever $f \in \mathrm{BV}(S)$. We make the inductive assumption that $\Delta_{n} f+\left(\circ ; x_{1}, \cdots, x_{n}\right) \geq 0$ for $f \in \mathrm{BV}(S)$ and $x_{j} \in X^{\prime}$. For $x_{n+1} \in X^{\prime}$, Lemma 4.5 implies 


$$
\begin{aligned}
\Delta_{n+1} f^{+}\left(\circ ; x_{1}, \cdots, x_{n+1}\right) & =\Delta_{n}\left[f^{+}-\left(f^{+}\right)_{x_{n+1}}\right]\left(\circ ; x_{1}, \ldots, x_{n}\right) \\
& =\Delta_{n}\left(f-f_{x_{n+1}}\right)^{+}\left(\circ ; x_{1}, \ldots, x_{n}\right) .
\end{aligned}
$$

But if $f \in \mathrm{BV}(S)$ then so does $f_{x_{n+1}}$ from Lemma 4.4 and hence Lemma $4.5 \mathrm{im}$ plies $\left(f-f_{x_{n+1}}\right) \in \mathrm{BV}(S)$. Therefore the inductive assumption and Lemma 4.6 shows $\Delta_{n+1} f^{+} \geq 0$ or $f^{+} \in C_{\infty}(S)$.

Remark. If we take $S=X^{\prime}$ in Lemma 4.6 then we see that $\left|f-f_{x}\right|=|f|-\left|f_{x}\right|$ for $f \in \mathrm{BV}(S)$. Moreover the proof of Theorem 4.1 implies $\left(f-f_{x}\right)^{ \pm}=f^{ \pm}-\left(f_{x}\right)^{ \pm}$ for $f \in \mathrm{BV}(S)$ and $|f| \geq(f)^{A}$ for all $f$.

5. Applications to a semilattice. Throughout $\$ 5, S$ will be a commutative idempotent semigroup (semilattice) with identity. As before, $\left(I_{1}\right)^{k}$ will be the set of all zero-one valued functions on the first $k$ natural numbers and for each $\tau \epsilon$ $\left(I_{1}\right)^{k}$ we will set $\bar{\tau}=\Sigma_{j=1}^{k} \tau(j)$. For $f \mid S \rightarrow R,\left\{x_{j} \mid j=1,2, \cdots, k\right\} \subset S$ and

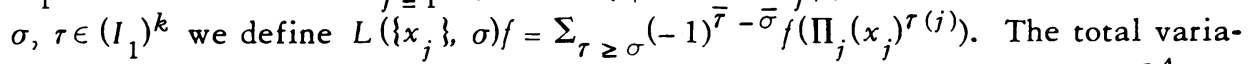
tion $\|f\|$ of $f$ has been defined by Newman [7] as $\sup _{\left\{x_{j}\right\}} \Sigma_{\sigma}\left[L\left(\left\{x_{j}\right\}, \sigma\right) f\right]^{A}$.

Theorem 5.1. If $f$ is a real-valued function on $S$ then $\|f\|$ and $\|f\|$ are botb equal to

$$
\sup _{\left\{x_{j}\right\}} \sum_{\sigma}\left[\Delta_{k-\bar{\sigma}} f\left(\prod_{\sigma(j)=1} x_{j} ;\left\{x_{j}\right\}_{\sigma(j)=0}\right)\right]^{A} .
$$

Proof. From $\$ 2$,

$$
\Delta_{k} f\left(x ; x_{1}, \cdots, x_{k}\right)=\sum_{m=0}^{k}(-1)^{m} \sum_{\sigma_{=}=m} f\left(x \cdot \prod_{j}\left(x_{j}\right)^{\sigma(j)}\right) .
$$

Thus

$$
\begin{aligned}
L\left(\left\{x_{j}\right\}, \sigma\right) f & =\sum_{m=0}^{k-\bar{\sigma}}(-1)^{k} \sum_{\bar{\tau}-\delta_{=m}} f\left[\left(\prod_{\sigma(j)=1} x_{j}\right)\left(\prod_{\tau(j)>\sigma(j)} x_{j}\right)\right] \\
& =\Delta_{k-\bar{\sigma}} f\left(\prod_{\sigma(j)=1} x_{j} ;\left\{x_{j}\right\}_{\sigma(j)=0}\right) .
\end{aligned}
$$

Equality of $\|f \mid\|$ and (i) is therefore established. The following two formulas easily follow since every element of $S$ is idempotent.

$$
\Delta_{n} f\left(x_{j} y_{1} x_{1}, \cdots, x_{j}, \cdots, x_{n}\right)=0,
$$

$$
\Delta_{n+1} f\left(x ; x_{1}, \cdots, x_{n}, x_{n}\right)=\Delta_{n} f\left(x ; x_{1}, \cdots, x_{n}\right) .
$$


From (ii) we see that

$$
\prod_{j}\left(\begin{array}{c}
n \\
i_{j}
\end{array}\right) \Delta f\left(\prod_{j}\left(x_{j}\right)^{i} j_{\frac{x_{1}}{n}, \cdots, x_{1}}, \cdots, \frac{x_{k}, \cdots, x_{k}}{n-i_{k}}\right)=0
$$

unless $i_{j}$ is 0 or $n$ for all $j$. It follows from (iii) that

$$
\sum\left\{\left[\Delta /\left(n,\left\{x_{j}\right\}, i\right)\right]^{A} \mid i \in\left(I_{n}\right)^{k}\right\}=\sum\left\{\left[\Delta f\left(1,\left\{x_{j}\right\}, i\right)\right]^{A} \mid i \in\left(I_{1}\right)^{k}\right\} .
$$

Since this latter summation is nothing more than

$$
\sum_{\sigma}\left[\Delta_{k-\bar{\sigma}} f\left(\prod_{\sigma(j)=1} x_{j} ;\left\{x_{j} \mid \sigma(j)=0\right\}\right)\right]^{A},
$$

Lemma 4.3 (ii) implies (i) agrees with $\|$ o .

Remarks. (a) The work of $\oint_{4}$ shows that $\sup _{\left\{x_{j}\right\}}$ may be replaced by $\sup _{X}$ where $X$ is any finite subset of distinct elements of an arbitrary but fixed generator set $X^{\prime}$. Moreover $\sup _{X}$ may be further replaced by $\lim _{X}$.

(b) If $S$ is a linearly ordered set and $x y$ is defined by $x \wedge y$ then $(S, \wedge)$ is a semilattice. For this case $\Delta_{k} f\left(x ; x_{1}, \cdots, x_{k}\right)=\Delta_{1} f\left(x ; x_{1} \vee \cdots \vee x_{k}\right)$ so that a typical term of the summation in (i) looks like

$$
\left[\Delta_{1} f\left(\underset{\sigma(j)=1}{\wedge}\left(x_{j}\right) ; \underset{\sigma(j)=0}{\bigvee}\left(x_{j}\right)\right)\right]^{A}
$$

Now if $x_{j} \leq x_{l}$ for some $j, l$ with $\sigma(j)=1$ and $\sigma(l)=0$ then $\bigwedge_{\sigma(j)=1}\left(x_{j}\right) \leq$ $V_{\sigma(j)}=0\left(x_{j}\right)$ so that (iv) is zero. Without loss of generality we may assume $x_{1} \leq \cdots \leq x_{k}$. Then (i) reduces to $\sup \left\{x_{j}\right\}\left\{\Sigma_{j=1}^{k-1}\left[f\left(x_{j+1}\right)-f\left(x_{j}\right)\right]^{A}+\left[f\left(x_{1}\right)\right]^{A}\right\}$ which the reader will recognize as the classical definition of total variation of a function on a linearly ordered set.

A formally different notion of total variation of functions on a semilattice was introduced in [4]. However, the BV-functions of [4] were seen to be the functions in $E_{\infty}(S)$ and the total variation in that sense of such a function was also seen to be $\int_{\exp s} e(\circ) d\left|\mu_{f}\right|$. Thus both of these notions must be equivalent. If $S$ is a distributive lattice with $\wedge$ as its semigroup operation then the results of [4] also show that Birkhoff's notion [1, p. 83] of total variation of valuations agrees with that introduced here.

6. Applications to moment sequences. Since a generator set for $(N,+)$ is $\{1\}$, Theorem 4.1 offers a new proof of the following classical result of Hausdorff (cf. $[10$, p. 103]).

Corollary 6.1. A sequence $f \mid N \rightarrow R$ is a moment sequence if and only if 


$$
\|f\|=\sup _{n} \sum_{j}\left(\begin{array}{l}
n \\
j
\end{array}\right)\left[\Delta_{n-j} f(j ; 1, \cdots, 1)\right]^{A}<\infty .
$$

Of course Theorem 4.1 gives an immediate and obvious generalization to Corollary 6.1 for the case where $S$ is a product of $k$-copies of $N$. In this event $S$ has $k$ generators.

Let $R^{+}$denote the additive semigroup of nonnegative real numbers. It is interesting to note that the total variation of a function $f \in \mathrm{BV}\left(R^{+}\right)$is also given by (i). To see this we note that $\exp (N)=\exp \left(R^{+}\right)$. Thus every moment sequence $f$ has a unique extension to a real-valued function $\bar{f}$ on $R^{+}$with the same representing measure $\mu_{f}$. The claim follows since $\|f\|=\left\|\mu_{f}\right\|=\|\bar{f}\|$. However it is not true that a function $f$ on $R^{+}$is in $\mathrm{BV}\left(R^{+}\right)$whenever the supremum in (i) is finite. Indeed that supremum only reflects values of $f$ in the range of $N$ and a moment sequence has only one extension to a function in $\mathrm{BV}\left(R^{+}\right)$.

7. The lattice algebra $\mathrm{BV}(S)$. Other properties of $\mathrm{BV}(S)$ ( $S$ a commutative semigroup with identity) can easily be derived from the results of [3] which we feel should be mentioned here. First of all the convolution $\mu * \nu$ of two regular Borel measures $\mu$ and $\nu$ on the compact semigroup $\exp (S)$ can be defined in the usual way. The following two propositions follow easily from [3].

Proposition 6.1. If $f, g \in \mathrm{BV}(S)$ then $\mu_{f} * \mu_{g}=\mu_{f g}$. In particular BV $(S)$ is a Banacb algebra under pointwise multiplication.

Proposition 6.2. $\mathrm{BV}(S)$ is a Banach lattice whose positive cone is the cone of completely monotonic functions.

Remark. Thus $f \in \mathrm{BV}(S)$ if and only if $f$ is the difference of two completely monotonic functions. Since the completely monotonic functions on a linearly ordered set (regarding this set as a semilattice under $\wedge$ ) are the nonnegative nondecreasing functions, we get the well-known decomposition of the classical BV-functions into monotonic functions.

\section{REFERENCES}

1. G. Birkhoff, Lattice theory, Amer. Math. Soc. Colloq. Publ., vol. 25, Amer. Math. Soc., Providence, R. I., 1948. MR 10, 673.

2. N. Dunford and J. T. Schwartz, Linear operators. I: General theory, Pure and Appl. Math., vol. 7, Interscience, New York, 1958. MR 22 \#8302.

3. N. J. Fine and P. H. Maserick, On the simplex of completely monotonic functions on a commutative semigroup, Canad. J. Math. 22 (1970), 317-326. MR 41 \#2338.

4. J. Kist and P. H. Maserick, BV-functions on semilattices, Pacific J. Math. 37 (1971), 711-723.

5. G. Lorentz, Bernstein polynomials, Math. Expositions, no. 8, Univ. of Toronto Press, Toronto, 1953. MR 15, 217. 
6. S. E. Newman, Measure algebras and functions of bounded variation on idempotent semigroups, Bull. Amer. Math. Soc. 75 (1969), 1396-1400. MR 40 \#478

7. - Measure algebras and functions of bounded variation on idempotent semigroups, Trans. Amer. Math. Soc. 163 (1972), 189-205.

8. A. L. Peressini, Ordered topological vector spaces, Harper \& Row, New York, 1967. MR 37 \#3315.

9. J. A. Shohat and J. D. Tamarkin, The problem of moments, Math. Surveys, no. 11, Amer. Math. Soc., Providence, R. I., 1943. MR 5, 5; MR 13, 1138.

10. D. V. Widder, The Laplace transform, Princeton Math. Series, vol. 6, Princeton Univ. Press, Princeton, N. J., 1941. MR 3, 232.

DEPARTMENT OF MATHEMATICS, PENNSYLVANIA STATE UNIVERSITY, UNIVERSITY PARK, PENNSYLVANIA 16802 